Pacific Journal of Mathematics

ISOMORPHISMS OF SPACES OF NORM-CONTINUOUS 


\title{
ISOMORPHISMS OF SPACES OF NORM-CONTINUOUS FUNCTIONS
}

\author{
Michael CAMBERN
}

\begin{abstract}
If $X$ and $Y$ are compact Hausdorff spaces and $E$ a uniformly convex Banach space, then the existence of an isomorphism $T$ of $C(X, E)$ onto $C(Y, E)$ with $\|T\|\left\|T^{-1}\right\|$ small implies that $X$ and $Y$ are homeomorphic.
\end{abstract}

1. Introduction. Throughout this article, the letters $X, Y, Z$, and $W$ will denote compact Hausdorff spaces, and $E$ a Banach space. $C(X, E)$ denotes the space of continuous functions on $X$ to $E$ provided with the supremum norm. If $E$ is a dual space then $C\left(X, E_{\sigma^{*}}\right)$ stands for the Banach space of continuous functions $F$ on $X$ to $E$ when this latter space is provided with its weak ${ }^{*}$ topology, again normed by $\|F\|_{\infty}=$ $\sup _{x \in X}\|F(x)\|$. If $E$ is the one-dimensional field of scalars then we write $C(X)$ for $C(X, E)$. The interaction between elements of a Banach space and those of its dual is denoted by $\langle\cdot, \cdot\rangle$. We write $E_{1} \cong E_{2}$ to indicate that the Banach spaces $E_{1}$ and $E_{2}$ are isometric.

The well known Banach-Stone theorem states that if $C(X)$ and $C(Y)$ are isometric then $X$ and $Y$ are homeomorphic. Various authors, beginning with $M$. Jerison [13], have considered the problem of determining geometric properties of $E$ which allow generalizations of this theorem to spaces of norm-continuous vector functions $C(X, E)$. The most exhaustive compilation of results of this nature can be found in the monograph by E. Behrends [2]. Another type of generalization of the theorem was obtained independently in [1] and [3], and, while still dealing with scalar functions, replaces isometries by isomorphisms $T$ with $\|T\|\left\|T^{-1}\right\|$ small.

The first attempt to combine these two directions of generalization is found in [4], where it is shown that if $E$ is a finite-dimensional Hilbert space, then the existence of an isomorphism $T$ of $C(X, E)$ onto $C(Y, E)$ with $\|T\|\left\|T^{-1}\right\|<\sqrt{2}$ implies that $X$ and $Y$ are homeomorphic. More recently, K. Jarosz [12] has obtained a similar generalization for Banach spaces $E$ whose dual space satisfies a geometric condition involving both $\|T\|\left\|T^{-1}\right\|$ and the number $4 / 3$. Here we obtain such a theorem for all uniformly convex spaces $E$. Moreover, given such a space $E$, the bound on the isomorphisms for which our theorem works depends on the modulus of convexity associated with $E$. 
Our method of proof depends on a characterization of the second dual space of $C(X, E)$, and is analogous to the method used by $\mathrm{H}$. B. Cohen in the scalar case to obtain a new proof of the results of [1] and [3]. The first dual of $C(X)$ is, of course, given by the Riesz representatioin theorem which states that $C(X)^{*}$ consists of all finite, regular, scalar-valued Borel measures $\mu$ on $X$. The vector analogue of this result was obtained by $\mathrm{I}$. Singer in [15], where it is shown that $C(X, E)^{*}$ is the Banach space of all regular Borel measures $m$ on $X$ to $E^{*}$, with finite variation $|m|$, and norm given by $\|m\|=|m|(X)$. An English version of the proof of this theorem can be found in [16, p. 192].

In [7] Cohen exploited the fact, first established by Kakutani [14], that $C(X)^{* *}$ is isometric to a space $C(Z)$ for a particular compact Hausdorff space $Z$ dependent on $X$. And in [5] it is shown that if $X$ is dispersed or if $E^{*}$ has the Radon-Nikodym property, then $C(X, E)^{* *} \cong C\left(Z, E_{\sigma^{*}}^{* *}\right)$ where $Z$ is that compact Hausdorff space such that $C(X)^{* *} \cong C(Z)$. The interaction between the elements of the first dual of $C(X, E)$ (that is, vector measures on $X)$, and functions in $C\left(Z, E_{\sigma^{*}}^{* *}\right)$ is given explicitly in [6]. It is the result of [5] on which we base most of our arguments.

We shall assume henceforth, that $E$ is a uniformly convex Banach space. Let $U$ denote the unit ball in $E$ and let

$$
\delta(\varepsilon)=\inf _{e_{1}, e_{2} \in U}\left\{1-\left\|\left(e_{1}+e_{2}\right) / 2\right\|:\left\|e_{1}-e_{2}\right\| \geq \varepsilon\right\} .
$$

Recall that $E$ is uniformly convex means that $\delta(\varepsilon)>0$ when $0<\varepsilon \leq 2$. We will frequently use the fact that we always have $\delta(1) \leq \frac{1}{2}$.

The uniform convexity of $E$ enters into our proof in a number of ways. First, we rely upon a geometric property of uniformly convex spaces which we establish in Lemma 1 . Also $E$ uniformly convex implies that $E$ is reflexive [8, p. 147], and thus $E^{*}$ has the Radon-Nikodym property [9, p. 218] and the result of [5] applies. We wish to prove the following:

THEOREM. Let $X$ and $Y$ be compact Hausdorff spaces and $E$ a uniformly convex Banach space. If $T$ is an isomorphism of $C(X, E)$ onto $C(Y, E)$ satisfying $\|T\|\left\|T^{-1}\right\|<(1-\delta(1))^{-1}$, then $X$ and $Y$ are homeomorphic.

The proof of the theorem will be established via a sequence of lemmas and a proposition. However we first note the following. By replacing $T$ by the isomorphism $(1+\varepsilon)\left\|T^{-1}\right\| T$ for a sufficiently small positive number $\varepsilon$, we may suppose, without loss of generality, that $T$ is strictly norm-increasing-i.e., $\|T F\|_{\infty} \geq(1+\varepsilon)\|F\|_{\infty}$, for $F \in C(X, E)$, and that we have $\|T\|<(1-\delta(1))^{-1}$. Fix such an $\varepsilon$, and then fix a positive number $P$ with 
$1<P<1+\varepsilon$. We will thus assume, throughout the remainder of this article, that we are dealing with an isomorphism $T$ of $C(X, E)$ onto $C(Y, E)$ satisfying $\|T F\|_{\infty}>P\|F\|_{\infty}$ for $F \in C(X, E), F \neq 0$ and $\|T\|<$ $(1-\delta(1))^{-1}$.

Since here we have $E^{* *}=E$, it follows that $C(X, E)^{* *}$ is of the form $C\left(Z, E_{\sigma^{*}}\right)$ for a certain compact Hausdorff space $Z$. Similarly, $C(Y, E)^{* *}$ $\cong C\left(W, E_{\sigma^{*}}\right)$ for that compact Hausdorff space $W$ with $C(Y)^{* *} \cong C(W)$. We can thus regard $T^{* *}$ as a strictly norm-increasing isomorphism of $C\left(Z, E_{\sigma^{*}}\right)$ onto $C\left(W, E_{\sigma^{*}}\right)$ satisfying $\left\|T^{* *}\right\|<(1-\delta(1))^{-1}$ and $\left\|T^{* *} F\right\|_{\infty}$ $>P\|F\|_{\infty}$ for $F \in C\left(Z, E_{\sigma^{*}}\right), F \neq 0$.

Next note that if $F^{*} \in C\left(Z, E_{\sigma^{*}}\right)^{*}$, then the restriction of $F^{*}$ to $C(Z, E)$ is a continuous linear functional of norm less than or equal to $\left\|F^{*}\right\|$. Thus, by Singer's result, this restriction is given by a regular Borel vector measure $n$ on $X$ to $E^{*}$ with $\|n\| \leq\left\|F^{*}\right\|$. If $z$ is any point of $Z, n$ can then be uniquely decomposed as $n=\psi \cdot \mu_{z}+m$, where $\mu_{z}$ denotes the scalar unit point mass at $z, \psi \in E^{*}$, and $m \in C(Z, E)^{*}$ with $m(\{z\})=0$. (Take $\psi=n(\{z\})$ and $m=n-\psi \cdot \mu_{z}$.) We then let $\bar{m}$ denote any normpreserving linear extension of $m$ to an element of $C\left(Z, E_{\sigma^{*}}\right)^{*}$ and set $=F^{*}-\psi \cdot \mu_{z}-\bar{m}$. Then $\Phi$ is a continuous linear functional on

$\left.Z, E_{\sigma^{*}}\right)$ which vanishes on $C(Z, E)$ and $F^{*}=\psi \cdot \mu_{z}+\bar{m}+\Phi$. Whenever we write an element $F^{*} \in C\left(Z, E_{\sigma^{*}}\right)^{*}$ in this manner, $F^{*}=$ $\psi \cdot \mu_{z}+\bar{m}+\Phi$, it will be implicit that $\psi \in E^{*}$, that $\bar{m}$ is a fixed HahnBanach extension of the vector measure $m$ determined as above, and consequently that $\Phi \in C(Z, E)^{\perp}$. A similar convention applies when we write an element $G^{*} \in C\left(W, E_{\sigma^{*}}\right)^{*}$ as $G^{*}=\psi \cdot \mu_{w}+\bar{m}+\Phi$.

Finally, we let $X_{0}$ denote the set of isolated points of $Z$. It is known that each point of $X_{0}$ is of the form $t x$ for some $x \in X$, where $t$ is the canonical (nontopological) injection of $X$ into $Z$, and every such point $t x$ is isolated [11, p. 841]. Similarly, we let $Y_{0}$ denote the set of isolated points of $W$ so that $Y_{0}$ consists of the points $s y, y \in Y$, where $s$ is the corresponding injection of $Y$ into $W$.

\section{Proof of the Theorem.}

LEMMA 1. If $E$ is a uniformly convex normed linear space and $r$ is a positive integer, and if we are given $2^{r}$ elements $e_{j} \in E$ with $\left\|e_{j}\right\| \geq \eta>0$ for $1 \leq j \leq 2^{r}$, then

(i) there exists scalars $\lambda_{j}, 1 \leq j \leq 2^{r}$, with $\left|\lambda_{j}\right| \leq 1$ for all $j$ such that $\left\|\sum_{j=1}^{2^{r}} \lambda_{j} e_{j} /\right\| e_{j}\|\| \geq(1-\delta(1))^{-r}$, and consequently

(ii) there exist scalars $\alpha_{j}, 1 \leq j \leq 2^{r}$, with $\left|\alpha_{j}\right| \leq 1$ for all $j$ such that $\left\|\sum_{j=1}^{2^{r}} \alpha_{j} e_{j}\right\| \geq \eta(1-\delta(1))^{-r}$. 
Proof. The proof is established by induction on $r$. First assume that $r=1$ and that $e_{1}, e_{2} \in E$, with $\left\|e_{j}\right\| \geq \eta, j=1,2$. Then

$$
e_{1} /\left\|e_{1}\right\|=\frac{1}{2}\left(e_{1} /\left\|e_{1}\right\|+e_{2} /\left\|e_{2}\right\|\right)+\frac{1}{2}\left(e_{1} /\left\|e_{1}\right\|-e_{2} /\left\|e_{2}\right\|\right) \text {, }
$$

and, since a uniformly convex space is strictly convex, we must thus have either

$$
\left\|e_{1} /\right\| e_{1}\left\|+e_{2} /\right\| e_{2}\|\|>1 \text { or }\left\|e_{1} /\right\| e_{1}\left\|-e_{2} /\right\| e_{2}\|\|>1
$$

and both of these norms are less than or equal to 2 . Let $M$ be the maximum of these two norms. Then by taking $\lambda_{1}=1$ and $\lambda_{2}=1$ or -1 we can find scalars $\lambda_{j}$ of modulus one such that

$$
\left\|\lambda_{1} e_{1} /\right\| e_{1}\left\|+\lambda_{2} e_{2} /\right\| e_{2}\|\|=M>1 \text {. }
$$

Now

$$
a=(1 / M)\left(\lambda_{1} e_{1} /\left\|e_{1}\right\|+\lambda_{2} e_{2} /\left\|e_{2}\right\|\right)
$$

and

$$
b=(1 / M)\left(\lambda_{1} e_{1} /\left\|e_{1}\right\|-\lambda_{2} e_{2} /\left\|e_{2}\right\|\right)
$$

are in the closed unit ball $U$ of $E$ and $(1 / M)\left(\lambda_{1} e_{1} /\left\|e_{1}\right\|\right)$ is the midpoint of the segment joining them. Also, since $\|a-b\|=2 / M$ and $M$ is less than or equal to 2, we have

$$
1-1 / M=1-\left\|(1 / M)\left(\lambda_{1} e_{1} /\left\|e_{1}\right\|\right)\right\| \geq \delta(2 / M) \geq \delta(1),
$$

giving $M \geq(1-\delta(1))^{-1}$ and establishing (i) for $r=1$.

Next let $N=\min \left\{\left\|e_{1}\right\|,\left\|e_{2}\right\|\right\}$. Then from $(*)$ we have

$$
\left\|\left(N \lambda_{1} /\left\|e_{1}\right\|\right) e_{1}+\left(N \lambda_{2} /\left\|e_{2}\right\|\right) e_{2}\right\|=N \cdot M \geq \eta(1-\delta(1))^{-1} .
$$

Thus letting $\alpha_{J}=N \lambda_{j} /\left\|e_{j}\right\|$ for $j=1,2$ we have established (ii) for $r=1$.

Now assume the lemma is valid for all $r$ with $1 \leq r \leq k$, and that we are given elements $e_{j} \in E, 1 \leq j \leq 2^{k+1}$, with $\left\|e_{j}\right\| \geq \eta$ for all $j$. By the inductive hypothesis there exist scalars $\hat{\lambda}_{j}, 1 \leq j \leq 2^{k+1}$, with $\left|\hat{\lambda}_{j}\right| \leq 1$ for all $j$ such that

$$
\left\|\sum_{j=1}^{2^{k}} \hat{\lambda}_{j} e_{j} /\right\| e_{j}\|\|=M_{1} \geq(1-\delta(1))^{-k}
$$

and

$$
\left\|\sum_{j=2^{k}+1}^{2^{k+1}} \hat{\lambda}_{j} e_{J} /\right\| e_{J}\|\|=M_{2} \geq(1-\delta(1))^{-k}
$$

Then

$$
c=\left(\frac{1}{M_{1}}\right) \sum_{j=1}^{2^{k}} \hat{\lambda}_{j} e_{j} /\left\|e_{j}\right\| \text { and } d=\left(\frac{1}{M_{2}}\right) \sum_{j=2^{k}+1}^{2^{k+1}} \hat{\lambda}_{j} e_{j} /\left\|e_{j}\right\|
$$


belong to $U$ and $c=\left(\frac{1}{2}\right)(c+d)+\left(\frac{1}{2}\right)(c-d)$. Since $\|c\|=1$, again we must have either $\|c+d\|>1$ or $\|c-d\|>1$, and both of these norms are $\leq 2$.

Let $M$ be the maximum of these two norms. Thus taking either $\tilde{\lambda}_{j}=\hat{\lambda}_{j}$ for all $j$ with $2^{k}+1 \leq j \leq 2^{k+1}$, or $\tilde{\lambda}_{j}=-\hat{\lambda}_{j}$ for all such $j$, we can find $\tilde{\lambda}_{j}$ with $\left|\tilde{\lambda}_{j}\right| \leq 1$ such that

$$
\left\|\left(\frac{1}{M_{1}}\right) \sum_{j=1}^{2^{k}} \hat{\lambda}_{j} e_{j} /\right\| e_{j}\left\|+\left(\frac{1}{M_{2}}\right) \sum_{j=2^{k}+1}^{2^{k+1}} \tilde{\lambda}_{j} e_{j} /\right\| e_{j}\|\|=M>1 .
$$

Let $e=\left(1 / M_{2}\right) \sum_{j=2^{k}+1}^{2^{k+1}} \tilde{\lambda}_{j} e_{j} /\left\|e_{j}\right\|$. Now $a=(1 / M)(c+e)$ and $b=$ $(1 / M)(c-e)$ are in $U$ and $(1 / M) c$ is the midpoint of the segment joining them. Also $\|a-b\|=2 / M$. Hence

$$
1-1 / M=1-\|(1 / M) c\| \geq \delta(2 / M) \geq \delta(1),
$$

giving $M \geq(1-\delta(1))^{-1}$.

Let $M_{0}=\min \left\{M_{1}, M_{2}\right\}$. Then from $\left({ }^{* *}\right)$ we have

$$
\left\|\sum^{\|} \sum^{2^{k}}\left(\frac{M_{0} \hat{\lambda}_{j}}{M_{1}}\right) \frac{e_{j}}{\left\|e_{j}\right\|}+\sum_{j=2^{k}+1}^{2^{k+1}}\left(\frac{M_{0} \tilde{\lambda}_{j}}{M_{2}}\right) \frac{e_{j}}{\left\|e_{j}\right\|}\right\|=M \cdot M_{0} \geq(1-\delta(1))^{-k-1},
$$

so that, by letting $\lambda_{j}=M_{0} \hat{\lambda}_{j} / M_{1}$ for $1 \leq j \leq 2^{k}$ and $\lambda_{j}=M_{0} \tilde{\lambda}_{j} / M_{2}$ for $2^{k}+1 \leq j \leq 2^{k+1}$, we have established (i) for $r=k+1$.

Finally let $N=\min \left\{\left\|e_{j}\right\|: j=1, \ldots, 2^{k+1}\right\}$. We then have

$$
\left\|\sum_{j=1}^{2^{k+1}}\left(\frac{N \lambda_{j}}{\left\|e_{j}\right\|}\right) e_{j}\right\| \geq N(1-\delta(1))^{-k-1} \geq \eta(1-\delta(1))^{-k-1}
$$

and thus, setting $\alpha_{j}=N \lambda_{j} /\left\|e_{j}\right\|$ for $1 \leq j \leq 2^{k+1}$, we have established (ii) for $r=k+1$. This completes the proof.

LEMma 2. If $w \in W$ and $t x \in X_{0}$ then there exists an element $\phi$ of $E^{*}$ with $\|\phi\|=1$ such that $T^{* * *} \phi \cdot \mu_{w}$ is of the form $\psi \cdot \mu_{t x}+\bar{m}+\Phi$ with $\|\psi\|>P$ if, and only if, for some $e \in E$ with $\|e\|=1$ we have $\left\|T^{* *}\left(\chi_{\{t x\}} \cdot e\right)(w)\right\|>P$.

Proof. Suppose that for some $e \in E$ with $\|e\|=1$ we have $\left\|T^{* *}\left(\chi_{\{t x\}} \cdot e\right)(w)\right\|>P$. Choose $\phi \in E^{*}$ with $\|\phi\|=1$ such that

$$
\left\langle T^{* *}\left(\chi_{\{t x\}} \cdot e\right)(w), \phi\right\rangle=\left\|T^{* *}\left(\chi_{\{t x\}} \cdot e\right)(w)\right\|
$$


Then writing $T^{* * *} \phi \cdot \mu_{w}$ as $\psi \cdot \mu_{t x}+\bar{m}+\Phi$ we would have

$$
\begin{aligned}
P & <\left\|T^{* *}\left(\chi_{\{t x\}} \cdot e\right)(w)\right\|=\left\langle T^{* *}\left(\chi_{\{t x\}} \cdot e\right)(w), \phi\right\rangle \\
& =\int T^{* *}\left(\chi_{\{t x\}} \cdot e\right) d\left(\phi \cdot \mu_{w}\right)=\left\langle\chi_{\{t x\}} \cdot e, T^{* * *} \phi \cdot \mu_{w}\right\rangle \\
& =\int\left(\chi_{\{t x\}} \cdot e\right) d\left(\psi \cdot \mu_{t x}+m\right)+\left\langle\chi_{\{t x\}} \cdot e, \Phi\right\rangle=\langle e, \psi\rangle,
\end{aligned}
$$

and hence $\|\psi\|>P$.

Conversely, suppose there exists a $\phi \in E^{*}$ with $\|\phi\|=1$ such that $T^{* * *} \phi \cdot \mu_{w}$ has the specified form. Take $e \in E$ with $\|e\|=1$ such that $\langle e, \psi\rangle>P$. A computation exactly like that above then gives

$$
\left\langle T^{* *}\left(\chi_{\{t x\}} \cdot e\right)(w), \phi\right\rangle=\langle e, \psi\rangle>P
$$

and, consequently, $\left\|T^{* *}\left(\chi_{\{t x\}} \cdot e\right)(w)\right\|>P$.

We now let $W_{1}$ denote the set of all $w \in W$ such that for some $\phi \in E^{*}$ with $\|\phi\|=1$ there exists a $t x \in X_{0}$ with $T^{* * *} \phi \cdot \mu_{w}=\psi \cdot \mu_{t x}+$ $\bar{m}+\Phi$, where $\|\psi\|>P$. Then define $\rho: W_{1} \rightarrow X_{0}$ by $\rho(w)=t x$ if $w$ and $t x$ are related as in the previous sentence.

We first note that $\rho$ is a well defined map from $W_{1}$ to $X_{0}$. For by Lemma 2 we have $w \in W_{1}$ and $\rho(w)=t x$ if, and only if, for some $e \in E$ with $\|e\|=1$ we have $\left\|T^{* *}\left(\chi_{\{t x\}} \cdot e\right)(w)\right\|>P$. Thus if we assume that there exist $\phi_{1}, \phi_{2} \in E^{*}$ with $\left\|\phi_{1}\right\|=\left\|\phi_{2}\right\|=1$ and

$$
T^{* * *} \phi_{i} \cdot \mu_{w}=\psi_{i} \cdot \mu_{t x_{i}}+\bar{m}_{i}+\Phi_{i}
$$

for $i=1,2$, with $\left\|\psi_{i}\right\|>P$ and $t x_{1} \neq t x_{2}$, then for all choices of scalars $\alpha_{i}$ with $\left|\alpha_{i}\right| \leq 1$ and all $e_{i} \in E$ with $\left\|e_{i}\right\|=1, i=1,2$, we would have $\left\|\alpha_{1} \chi_{\left\{t x_{1}\right\}} \cdot e_{1}+\alpha_{2} \chi_{\left\{t x_{2}\right\}} \cdot e_{2}\right\|_{\infty} \leq 1$. However, it follows from Lemmas 1 and 2 that for appropriate choices of $\operatorname{such} \alpha_{i}$ and $e_{i}$ we would have

$$
\begin{aligned}
& \left\|T^{* *}\left(\alpha_{1} \chi_{\left\{t x_{1}\right\}} \cdot e_{1}+\alpha_{2} \chi_{\left\{t x_{2}\right\}} \cdot e_{2}\right)\right\|_{\infty} \\
& \geq\left\|\alpha_{1} T^{* *}\left(\chi_{\left\{t x_{1}\right\}} \cdot e_{1}\right)(w)+\alpha_{2} T^{* *}\left(\chi_{\left\{t x_{2}\right\}} \cdot e_{2}\right)(w)\right\| \\
& \geq P(1-\delta(1))^{-1}>(1-\delta(1))^{-1},
\end{aligned}
$$

contradicting the fact that $\left\|T^{* *}\right\|<(1-\delta(1))^{-1}$. Consequently $\rho$ is well defined as claimed.

Moreover, $\rho$ maps $W_{1}$ onto $X_{0}$. For given $t x \in X_{0}$ then for any $e \in E$ with $\|e\|=1$ there exists some $w \in W$ such that $\left\|T^{* *}\left(\chi_{\{t x\}} \cdot e\right)(w)\right\|>P$. Thus, as noted in the second sentence of the previous paragraph, we have $w \in W_{1}$ and $\rho(w)=t x$. 
By arguments exactly analogous to those given above, one obtains the companion result:

LeMma 2'. If $z \in Z$ and sy $\in Y_{0}$ then there exists an element $\phi$ of $E^{*}$ with $\|\phi\|=1$ such that $T^{* * *-1} \phi \cdot \mu_{z}$ is of the form $\psi \cdot \mu_{s y}+\bar{m}+\Phi$ with $\|\psi\|>1-\delta(1)$ if, and only if, for some $e \in E$ with $\|e\|=1$ we have $\left\|T^{* *-1}\left(\chi_{\{s y\}} \cdot e\right)(z)\right\|>1-\delta(1)$.

We then let $Z_{1}$ denote the set of all $z \in Z$ such that for some $\phi \in E^{*}$ with $\|\phi\|=1$ there exists an $s y \in Y_{0}$ with $T^{* * *-1} \phi \cdot \mu_{z}=\psi \cdot \mu_{s y}+\bar{m}+\Phi$, where $\|\psi\|>1-\delta(1)$. And we define $\tau: Z_{1} \rightarrow Y_{0}$ by $\tau(z)=$ sy if $z$ and $s y$ are related as in the previous sentence. Just as before one establishes that $\tau$ is a well defined map carrying $Z_{1}$ onto $Y_{0}$. Moreover, by Lemma 2 , we have $z \in Z_{1}$ and $\tau(z)=s y$ if and only if for some $e \in E$ with $\|e\|=1$ we have $\left\|T^{* *-1}\left(\chi_{\{s y\}} \cdot e\right)(z)\right\|>1-\delta(1)$.

LeMma 3. (i) For each $t x \in X_{0}, \rho^{-1}(\{t x\})$ is a finite open set of points, and consequently $W_{1} \subset Y_{0}$.

(ii) For each sy $\in Y_{0}, \tau^{-1}(\{s y\})$ is a finite open set of points, and consequently $Z_{1} \subseteq X_{0}$.

Proof. Suppose $t x \in X_{0}$ and $w \in \rho^{-1}(\{t x\})$. Then there exists an $e_{w} \in E$ with $\left\|e_{w}\right\|=1$ such that $\left\|T^{* *}\left(\chi_{\{t x\}} \cdot e_{w}\right)(w)\right\|>P$. Let

$$
\hat{e}_{w}=T^{* *}\left(\chi_{\{t x\}} \cdot e_{w}\right)(w) /\left\|T^{* *}\left(\chi_{\{t x\}} \cdot e_{w}\right)(w)\right\|
$$

and take any continuous $g: W \rightarrow[0,1]$ such that $g(w)=1$. Then define $G \in C(W, E) \subseteq C\left(W, E_{\sigma^{*}}\right)$ by $G\left(w^{\prime}\right)=g\left(w^{\prime}\right) \cdot \hat{e}_{w}, w^{\prime} \in W$. Now

$$
\left\|G+T^{* *}\left(\chi_{\{t x\}} \cdot e_{w}\right)\right\|_{\infty} \geq\left\|G(w)+T^{* *}\left(\chi_{\{t x\}} \cdot e_{w}\right)(w)\right\|>1+P,
$$

so that

$$
\left\|T^{* *-1}(G)+\chi_{\{t x\}} \cdot e_{w}\right\|_{\infty}>(1+P)(1-\delta(1)) \geq(1+P) / 2 .
$$

Thus as $\left\|T^{* *-1}(G)\right\|_{\infty}<1$ we must have $\left\|T^{* *-1}(G)(t x)\right\|>(P-1) / 2$.

Now pick any element $\phi_{w} \in E^{*}$ with $\left\|\phi_{w}\right\|=1$ such that $\left\langle\hat{e}_{w}, \phi_{w}\right\rangle=1$. Then $w \in\left\{w^{\prime} \in W:\left|\left\langle T^{* *}\left(\chi_{\{t x\}} \cdot e_{w}\right)\left(w^{\prime}\right), \phi_{w}\right\rangle\right|>P\right\}$, and this set is open. Moreover, for any $w^{\prime}$ in this set, we have $\left\|T^{* *}\left(\chi_{\{t x\}} \cdot e_{w}\right)\left(w^{\prime}\right)\right\|>P$ and thus $w^{\prime}$ must belong to $\rho^{-1}(\{t x\})$. Hence fixing such elements $e_{w}$ and $\phi_{w}$ for each $w \in \rho^{-1}(\{t x\})$ we have

$$
\rho^{-1}(\{t x\})=\bigcup_{w \in \rho^{-1}(\{t x\})}\left\{w^{\prime} \in W:\left|\left\langle T^{* *}\left(\chi_{\{t x\}} \cdot e_{w}\right)\left(w^{\prime}\right), \phi_{w}\right\rangle\right|>P\right\},
$$

an open set. 
We now show that $\rho^{-1}(\{t x\})$ is a finite set. Suppose that $w_{k}, 1 \leq k \leq$ $2^{r}$, are elements of $\rho^{-1}(\{t x\})$. We have seen that for each $k$ we can find $G_{k} \in C\left(W, E_{\sigma^{*}}\right)$ with $\left\|G_{k}\right\|_{\infty}=1$ and $\left\|T^{* *-1}\left(G_{k}\right)(t x)\right\|>(P-1) / 2$. If we choose the $G_{k}$ to have pairwise disjoint supports, then for all scalars $\alpha_{k}, 1 \leq k \leq 2^{r}$, with $\left|\alpha_{k}\right| \leq 1$, we have $\left\|\Sigma_{k=1}^{2^{r}} \alpha_{k} G_{k}\right\|_{\infty} \leq 1$. But by Lemma 1(ii), we can choose the $\alpha_{k}$ such that

$$
\left\|\sum_{k=1}^{2^{r}} \alpha_{k} T^{* *-1}\left(G_{k}\right)(t x)\right\| \geq \frac{(P-1)(1-\delta(1))^{-r}}{2} .
$$

Hence $\rho^{-1}(\{t x\})$ must be finite as claimed.

Thus for each $t x \in X_{0}, \rho^{-1}(\{t x\})$ is a finite open set of points, and thus consists entirely of isolated points. Hence $W_{1}=\bigcup_{t x \in X_{0}} \rho^{-1}(\{t x\})$ consists of isolated points and so $W_{1} \subseteq Y_{0}$, proving (i). The proof of (ii) is analogous.

LEMMA 4. Given an element of $C\left(Z, E_{\sigma^{*}}\right)^{*}$ of the form $\psi \cdot \mu_{t x}+\bar{m}+\Phi$, where tx $\in X_{0}$ is an isolated point of $Z$, then

$$
\left\|\psi \cdot \mu_{t x}+\bar{m}+\Phi\right\|=\|\psi\|+\|\bar{m}+\Phi\| \text {. }
$$

Proof. Suppose $\varepsilon>0$ is given. Choose $F \in C\left(Z, E_{\sigma^{*}}\right)$ with $\|F\|_{\infty} \leq 1$ such that $\langle F, \bar{m}+\Phi\rangle$ is real and greater than $\|\bar{m}+\Phi\|-\varepsilon$. Let $e_{1}=F(t x)$. Then both $\bar{m}$ and $\Phi$ annihilate $e_{1} \cdot \chi_{\{t x\}}$ so that $\left\langle F-e_{1} \chi_{\{t x\}}, \bar{m}+\Phi\right\rangle>\|\bar{m}+\Phi\|-\varepsilon$. Choose an element $e_{2} \in E$ with $\left\|e_{2}\right\|=1$ and $\left\langle e_{2}, \psi\right\rangle=\|\psi\|$. Then $\left\|F+\left(e_{2}-e_{1}\right) \cdot \chi_{\{t x\}}\right\|_{\infty} \leq 1$ and thus

$\left\|\psi \cdot \mu_{t x}+\bar{m}+\Phi\right\|$

$$
\begin{aligned}
& \geq\left|\left\langle F+\left(e_{2}-e_{1}\right) \cdot \chi_{\{t x\}}, \psi \cdot \mu_{t x}+\bar{m}+\Phi\right\rangle\right| \\
& =\int e_{2} \cdot \chi_{\{t x\}} d\left(\psi \cdot \mu_{t x}\right)+\left\langle F-e_{1} \cdot \chi_{\{t x\}}, \bar{m}+\Phi\right\rangle \\
& >\|\psi\|+\|\bar{m}+\Phi\|-\varepsilon .
\end{aligned}
$$

LEMMA 5. If sy $\in W_{1} \subseteq Y_{0}$ and $\rho(s y)=t x$, then $t x \in Z_{1}$ and $\tau(t x)=$ sy.

Proof. Let sy belong to $W_{1}$ and let $\rho(s y)=t x$. Suppose that either $t x$ is not an element of $Z_{1}$, or that $t x \in Z_{1}$, but $\tau(t x) \neq s y$. Either supposition leads to the conclusion that for all $e \in E$ with $\|e\|=1$ we have $\left\|T^{* *-1}\left(\chi_{\{s y\}} \cdot e\right)(t x)\right\| \leq 1-\delta(1)$.

Fix an $e \in E$ with $\|e\|=1$ and let $Q=\sup _{z \in Z}\left\|T^{* *-1}\left(\chi_{\{s y\}} \cdot e\right)(z)\right\|$. Then by Lemma 3(ii), and the paragraph preceding the statement of 
Lemma 3, we have

$$
\begin{aligned}
& \left\{z \in Z:\left\|T^{* *-1}\left(\chi_{\{s y\}} \cdot e\right)(z)\right\|>1-\delta(1)\right\} \\
& \quad=\left\{t x^{\prime} \in X_{0}:\left\|T^{* *-1}\left(\chi_{\{s y\}} \cdot e\right)\left(t x^{\prime}\right)\right\|>1-\delta(1)\right\} \subseteq \tau^{-1}(\{s y\}),
\end{aligned}
$$

a finite set, and thus we can find a $t x^{\prime} \in X_{0}$ such that

$$
\left\|T^{* *-1}\left(\chi_{\{s y\}} \cdot e\right)\left(t x^{\prime}\right)\right\|=Q \text {. }
$$

Now $t x^{\prime} \neq t x$ since $\tau(t x) \neq s y$.

Let $\hat{e}=T^{* *-1}\left(\chi_{\{s y\}} \cdot e\right)\left(t x^{\prime}\right)$ and $\tilde{e}=\hat{e} /\|\hat{e}\|$. Then consider the element $\chi_{\left\{t x^{\prime}\right\}} \cdot \tilde{e}$ of $C(Z, E) \subseteq C\left(Z, E_{\sigma^{*}}\right)$. There exists a $w \in W$ such that $\left\|T^{* *}\left(\chi_{\left\{t x^{\prime}\right\}} \cdot \tilde{e}\right)(w)\right\|>P$. Hence this $w$ belongs to $W_{1} \subseteq Y_{0}$ so $w=s y^{\prime}$ for some $s y^{\prime} \in Y_{0}$. Moreover $s y^{\prime} \neq s y$ since $\rho\left(s y^{\prime}\right)=t x^{\prime} \neq t x=\rho(s y)$.

From the proof of Lemma 2, we know that if $\phi \in E^{*}$ with $\|\phi\|=1$ is such that

$$
\left\langle T^{* *}\left(\chi_{\left\{t x^{\prime}\right\}} \cdot \tilde{e}\right)\left(s y^{\prime}\right), \phi\right\rangle=\left\|T^{* *}\left(\chi_{\left\{t x^{\prime}\right\}} \cdot \tilde{e}\right)\left(s y^{\prime}\right)\right\|
$$

then

$$
T^{* * *} \phi \cdot \mu_{s y^{\prime}}=\psi \cdot \mu_{t x^{\prime}}+\bar{m}+\Phi \quad \text { where }\langle\tilde{e}, \psi\rangle>P
$$

Hence $\langle\hat{e}, \psi\rangle=\|\hat{e}\|\langle\tilde{e}, \psi\rangle>Q P>Q$. We have

$$
\begin{aligned}
0 & =\int \chi_{\{s y\}} \cdot e d\left(\phi \cdot \mu_{s y^{\prime}}\right)=\left\langle\chi_{\{s y\}} \cdot e, \phi \cdot \mu_{s y^{\prime}}\right\rangle \\
& =\left\langle T^{* *-1}\left(\chi_{\{s y\}} \cdot e\right), T^{* * *} \phi \cdot \mu_{s y^{\prime}}\right\rangle \\
& =\int T^{* *-1}\left(\chi_{\{s y\}} \cdot e\right) d\left(\psi \cdot \mu_{t x^{\prime}}\right)+\left\langle T^{* *-1}\left(\chi_{\{s y\}} \cdot e\right), \bar{m}+\Phi\right\rangle \\
& =\langle\hat{e}, \psi\rangle+\left\langle T^{* *-1}\left(\chi_{\{s y\}} \cdot e\right), \bar{m}+\Phi\right\rangle .
\end{aligned}
$$

But the modulus of the first term on the right is greater than $Q$ while, by Lemma 4, the modulus of the second term on the right is less than or equal to $(\|T\|-\|\psi\|) Q<Q$. This contradiction completes the proof of the lemma.

Note that Lemma 5 implies that $X_{0}=\rho\left(W_{1}\right) \subseteq Z_{1}$, so that $X_{0}=Z_{1}$. It also shows that $Y_{0}=\tau\left(Z_{1}\right) \subseteq W_{1}$. For $\rho$ maps $W_{1}$ onto $X_{0}$; hence, given $t x \in Z_{1}=X_{0}$ there exists an $s y \in W_{1}$ with $\rho(s y)=t x$. And by Lemma 5 $\tau(t x)=s y \in W_{1}$. Thus $\rho$ maps $Y_{0}$ onto $X_{0}, \rho$ is injective since $\tau$ is a function and $\tau=\rho^{-1}$. It follows that $\hat{\rho}=t^{-1} \circ \rho \circ s$ is a one-one map of $Y$ onto $X$. We would like to show that $\hat{\rho}$ is a homeomorphism.

To this end again recall that we have $s y \in W_{1}=Y_{0}$ and $\rho(s y)=t x$ if, and only if, for some $e \in E$ with $\|e\|=1$ we have $\left\|T^{* *}\left(\chi_{\{t x\}} \cdot e\right)(s y)\right\|>P$. Since for any $e \in E$ with $\|e\|=1$ we must have $\left\|T^{* *}\left(\chi_{\{t x\}} \cdot e\right)(w)\right\|>P$ 
for some $w \in W$, it now follows that for all $e \in E$ with $\|e\|=1$ the only candidate for this $w$ is $s y$. That is, given $t x \in X_{0}$ let $s y=\tau(t x)$. Then for each $e \in E$ with $\|e\|=1$ we must have $\left\|T^{* *}\left(\chi_{\{t x\}} \cdot e\right)(s y)\right\|>P$ and $s y$ is the only point of $W$ for which such an inequality holds.

Next note that for $e \in E, \phi \in E^{*}, t x \in X_{0}$ and $s y \in Y_{0}$ we have

$$
\left\langle T^{* *}\left(\chi_{\{t x\}} \cdot e\right), \phi \cdot \mu_{s y}\right\rangle=\left\langle\phi \cdot \mu_{y}, T^{* *}\left(\chi_{\{t x\}} \cdot e\right)\right\rangle,
$$

the equality holding by the proof of Theorem 2 in [6]. We next have

$$
\left\langle\phi \cdot \mu_{y}, T^{* *}\left(\chi_{\{t x\}} \cdot e\right)\right\rangle=\left\langle T^{*}\left(\phi \cdot \mu_{y}\right), \chi_{\{t x\}} \cdot e\right\rangle
$$

by definition of the adjoint map, and then

$$
\left\langle T^{*}\left(\phi \cdot \mu_{y}\right), \chi_{\{t x\}} \cdot e\right\rangle=\left\langle e,\left(T^{*} \phi \cdot \mu_{y}\right)(\{x\})\right\rangle,
$$

again by the proof of Theorem 2 in [6]. Thus

$$
\left\langle T^{* *}\left(\chi_{\{t x\}} \cdot e\right), \phi \cdot \mu_{s y}\right\rangle=\left\langle e,\left(T^{*} \phi \cdot \mu_{y}\right)(\{x\})\right\rangle \text {. }
$$

Proposition. $\hat{\rho}$ is a homeomorphism of $Y$ onto $X$.

Proof. As noted above we have $\hat{\rho}(y)=x$ if, and only if, for all $e \in E$ with $\|e\|=1$ we have $\left\|T^{* *}\left(\chi_{\{t x\}}\right)(s y)\right\|>P$, which will be true if, and only if, for every $e$ there exists a $\phi \in E^{*}$ (depending on $e$ and $y$ ) with $\|\phi\|=1$ such that $\left\langle T^{* *}\left(\chi_{\{t x\}} \cdot e\right), \phi \cdot \mu_{s y}\right\rangle=\left\langle e,\left(T^{*} \phi \cdot \mu_{y}\right)(\{x\})\right\rangle$ is real and greater than $P$.

Now suppose that $\left\{y_{\beta}: \beta \in B\right\}$ is a net in $Y, y_{\beta} \rightarrow y_{0}$ but $x_{\beta}=\hat{\rho}\left(y_{\beta}\right)$ $\nrightarrow \hat{\rho}\left(y_{0}\right)=x_{0}$. Then there exists a compact neighborhood $V$ of $x_{0}$ such that for all $\beta_{0} \in B$ there is a $\beta \geq \beta_{0}$ with $x_{\beta}$ outside $V$.

Fix an $e \in E$ with $\|e\|=1$. By the paragraph before last there is a $\phi_{0} \in E^{*}$ with $\left\|\phi_{0}\right\|=1$ and $\left\langle e,\left(T^{*} \phi_{0} \cdot \mu_{y_{0}}\right)(\{x\})\right\rangle>P$. Write $T^{*} \phi_{0} \cdot \mu_{y_{0}}$ as $\psi_{0} \cdot \mu_{x_{0}}+m$, where $\psi_{0} \in E^{*}$ and $m$ is a regular Borel vector measure on $X$ to $E^{*}$ with $m\left(\left\{x_{0}\right\}\right)=0$. Then $\left\langle e, \psi_{0}\right\rangle>P$. Choose a neighborhood $V_{1}$ of $x_{0}, V_{1} \subseteq V$, such that $|m|\left(V_{1}\right)<P-1$. Next choose a continuous function $f_{1}: X \rightarrow[0,1]$ such that the support of $f_{1}$ is contained in $V_{1}$ and $f_{1}\left(x_{0}\right)=1$. Then define $F_{1} \in C(X, E)$ by $F_{1}(x)=f_{1}(x) \cdot e, x \in X$. We have

$$
\begin{aligned}
\left|\left\langle\left(T F_{1}\right)\left(y_{0}\right), \phi_{0}\right\rangle\right| & =\left|\left\langle\left(T F_{1}\right), \phi_{0} \cdot \mu_{y_{0}}\right\rangle\right|=\left|\left\langle F_{1}, T^{*}\left(\phi_{0} \cdot \mu_{y_{0}}\right)\right\rangle\right| \\
& =\left|\left\langle F_{1}, \psi_{0} \cdot \mu_{x_{0}}+m\right\rangle\right|=\left|\left\langle F_{1}\left(x_{0}\right), \psi_{0}\right\rangle+\int F_{1} d m\right| \\
& \geq\left\langle e, \psi_{0}\right\rangle-\int\left\|F_{1}\right\| d|m|>1 .
\end{aligned}
$$

Thus $\left\|\left(T F_{1}\right)\left(y_{0}\right)\right\|>1$. 
Since $y_{\beta} \rightarrow y_{0}$ and $T F_{1}$ is continuous in the norm topology, there is a $\beta_{0} \in B$ such that $\beta \geq \beta_{0}$ implies $\left\|\left(T F_{1}\right)\left(y_{\beta}\right)\right\|>1$. Thus fix a $\beta$ such that $\left\|\left(T F_{1}\right)\left(y_{\beta}\right)\right\|>1$ and $x_{\beta}=\hat{\rho}\left(y_{\beta}\right)$ lies outside $V$. Then for some $\phi_{\beta} \in E^{*}$ with $\left\|\phi_{\beta}\right\|=1$ we have $\left\langle e,\left(T^{*} \phi_{\beta} \cdot \mu_{y_{\beta}}\right)\left(\left\{x_{\beta}\right\}\right)\right\rangle>P$. Write $T^{*} \phi_{\beta} \cdot \mu_{y_{\beta}}$ as $\psi_{\beta} \cdot \mu_{x_{\beta}}+n$ where $\psi_{\beta} \in E^{*}$ and $n\left(\left\{x_{\beta}\right\}\right)=0$. Then $\left\langle e, \psi_{\beta}\right\rangle>P$. Take a neighborhood $V_{2}$ of $x_{\beta}$ disjoint from $V$ with $|n|\left(V_{2}\right)<P-1$ and choose continuous $f_{2}: X \rightarrow[0,1]$ such that the support of $f_{2}$ is contained in $V_{2}$ and $f_{2}\left(x_{\beta}\right)=1$. If we then define $F_{2} \in C(X, E)$ by $F_{2}(x)=f_{2}(x) \cdot e, x \in X$, it follows as above that $\left\|\left(T F_{2}\right)\left(y_{\beta}\right)\right\|>1$.

Now since $F_{1}$ and $F_{2}$ have disjoint supports, for every choice of scalars $\alpha_{i}$ with $\left|\alpha_{i}\right| \leq 1, i=1,2$, we have $\left\|\alpha_{1} F_{1}+\alpha_{2} F_{2}\right\|_{\infty} \leq 1$. However, by Lemma 1, there exist such scalars $\alpha_{i}$ with

$$
\left\|T\left(\alpha_{1} F_{1}+\alpha_{2} F_{2}\right)\right\|_{\infty} \geq\left\|\alpha_{1}\left(T F_{1}\right)\left(y_{\beta}\right)+\alpha_{2}\left(T F_{2}\right)\left(y_{\beta}\right)\right\|>(1-\delta(1))^{-1},
$$

which contradicts our assumptions about the norm of $T$. Thus $\hat{\rho}$ is a continuous, one-one map of $Y$ onto $X$, and is hence a homeomorphism.

\section{REFERENCES}

[1] D. Amir, On isomorphisms of continuous function spaces, Israel J. Math., 3 (1965), 205-210.

[2] E. Behrends, M-structure and the Banach-Stone Theorem, Lecture Notes in Mathematics 736, Springer-Verlag, Berlin-Heidelberg-New York, 1979.

[3] M. Cambern, On isomorphisms with small bound, Proc. Amer. Math. Soc., 18 (1967), 1062-1066.

[4] , Isomorphisms of spaces of continuous vector-valued functions, Illinois J. Math., 20 (1976), 1-11.

[5] M. Cambern and P. Greim, The bidual of $C(X, E)$, Proc. Amer. Math. Soc., 85 (1982), 53-58.

[6] _ـ The dual of a space of vector measures, Math. Z., 180 (1982), 373-378.

[7] H. B. Cohen, A second-dual method for $C(X)$ isomorphisms, J. Funct. Anal., 23 (1976), 107-118.

[8] M. M. Day, Normed Linear Spaces, 3rd. ed., Springer, Berlin-Heidelberg-New York, 1973.

[9] J. Diestel and J. J. Uhl, Jr., Vector measures, Math. Surveys 15, Amer. Math. Soc., Providence, R. I., 1977.

[10] N. Dinculeanu, Vector Measures, Pergamon Press, New York, 1967.

[11] $\mathrm{H}$. Gordon, The maximal ideal space of a ring of measurable functions, Amer. J. Math., 88 (1966), 827-843.

[12] K. Jarosz, A generalization of the Banach-Stone theorem, Studia Math., 73 (1982), 33-39.

[13] M. Jerison, The space of bounded maps into a Banach space, Ann. of Math., (2) 52 (1950), 309-327.

[14] S. Kakutani, Concrete representation of abstract (M)-spaces, Ann. of Math., (2) 42 (1941), 994-1024. 
[15] I. Singer, Linear functionals on the space of continuous mappings of a compact space into a Banach space (Russian), Rev. Roumaine Math. Pures Appl., 2 (1957), 301-315.

[16] _ Best Approximation in Normed Linear Spaces by Elements of Linear Subspaces, Springer-Verlag, Berlin and New York, 1970.

Received September 9, 1983

UNIVERSITY OF CALIFORNIA

SANTA BARbara, CA 93106 


\title{
PACIFIC JOURNAL OF MATHEMATICS EDITORS
}

\author{
DONALD BABBITT (Managing Editor) \\ University of California \\ Los Angeles, CA 90024 \\ J. DugundJI \\ University of Southern California \\ Los Angeles, CA 90089-1113 \\ R. FINN \\ Stanford University \\ Stanford, CA 94305 \\ Hermann FlaschKa \\ University of Arizona \\ Tucson, AZ 85721
}

\author{
C. C. MOORE \\ University of California \\ Berkeley, CA 94720 \\ ARTHUR OGUS \\ University of California \\ Berkeley, CA 94720 \\ Hugo Rossi \\ University of Utah \\ Salt Lake City, UT 84112 \\ H. SAMELSON \\ Stanford University \\ Stanford, CA 94305
}

\begin{tabular}{|c|c|c|c|c|}
\hline \multicolumn{5}{|c|}{ ASSOCIATE EDITORS } \\
\hline R. ARENS & $\begin{array}{l}\text { E. F. BECKENBACH } \\
(1906-1982)\end{array}$ & NeumanN & F. Wolf & K. YosHIDA \\
\hline \multicolumn{5}{|c|}{ SUPPORTING INSTITUTIONS } \\
\hline \multirow{2}{*}{\multicolumn{2}{|c|}{$\begin{array}{l}\text { UNIVERSITY OF ARIZONA } \\
\text { UNIVERSITY OF BRITISH COLUMBIA }\end{array}$}} & \multicolumn{3}{|c|}{ UNIVERSITY OF OREGON } \\
\hline & & \multirow{2}{*}{\multicolumn{3}{|c|}{ UNIVERSITY OF SOUTHERN CALIFORNIA }} \\
\hline \multirow{2}{*}{\multicolumn{2}{|c|}{$\begin{array}{l}\text { CALIFORNIA INSTITUTE OF TECHNOLOGY } \\
\text { UNIVERSITY OF CALIFORNIA }\end{array}$}} & & & \\
\hline & & \multicolumn{3}{|c|}{$\begin{array}{l}\text { STANFORD UNIVERSITY } \\
\text { UNIVERSITY OF HAWAII }\end{array}$} \\
\hline & \multicolumn{3}{|c|}{ UNIVER SITY OF TOKYO } \\
\hline \multicolumn{2}{|c|}{$\begin{array}{l}\text { MONTANA STATE UNIVERSITY } \\
\text { UNIVERSITY OF NEVADA, RENO }\end{array}$} & \multicolumn{3}{|l|}{ UNIVER } \\
\hline \multicolumn{2}{|c|}{ NEW MEXICO STATE UNIVERSITY } & \\
\hline \multicolumn{2}{|c|}{ OREGON STATE UNIVERSITY } & \multicolumn{3}{|c|}{$\begin{array}{l}\text { WASHINGTON STATE UNIVERSITY } \\
\text { UNIVERSITY OF WASHINGTON }\end{array}$} \\
\hline
\end{tabular}

The Supporting Institutions listed above contribute to the cost of publication of this Journal, but they are not owners or publishers and have no responsibility for its content or policies.

\begin{abstract}
Mathematical papers intended for publication in the Pacific Journal of Mathematics should be in typed form or offset-reproduced (not dittoed), double spaced with large margins. Please do not use built up fractions in the text of the manuscript. However, you may use them in the displayed equations. Underline Greek letters in red, German in green, and script in blue. The first paragraph must be capable of being used separately as a synopsis of the entire paper. In particular it should contain no bibliographic references. Please propose a heading for the odd numbered pages of less than 35 characters. Manuscripts, in triplicate, may be sent to any one of the editors. Please classify according to the scheme of Math. Reviews, Index to Vol. 39. Supply name and address of author to whom proofs should be sent. All other communications should be addressed to the managing editor, or Elaine Barth, University of California, Los Angeles, California 90024.

There are page-charges associated with articles appearing in the Pacific Journal of Mathematics. These charges are expected to be paid by the author's University, Government Agency or Company. If the author or authors do not have access to such Institutional support these charges are waived. Single authors will receive 50 free reprints; joint authors will receive a total of 100 free reprints. Additional copies may be obtained at cost in multiples of 50 .
\end{abstract}

The Pacific Journal of Mathematics is issued monthly as of January 1966. Regular subscription rate: $\$ 190.00$ a year (5 Vols., 10 issues). Special rate: $\$ 66.00$ a year to individual members of supporting institutions.

Subscriptions, orders for numbers issued in the last three calendar years, and changes of address should be sent to Pacific Journal of Mathematics, P.O. Box 969, Carmel Valley, CA 93924, U.S.A. Old back numbers obtainable from Kraus Periodicals Co., Route 100, Millwood, NY 10546.

The Pacific Journal of Mathematics at P.O. Box 969, Carmel Valley, CA 93924 (ISSN 0030-8730) publishes 5 volumes per year. Application to mail at Second-class postage rates is pending at Carmel Valley, California, and additional mailing offices. Postmaster: Send address changes to Pacific Journal of Mathematics, P.O. Box 969, Carmel Valley, CA 93924.

\section{PUBLISHED BY PACIFIC JOURNAL OF MATHEMATICS, A NON-PROFIT CORPORATION}




\section{Pacific Journal of Mathematics}

\section{Vol. 116, No. 2 December, 1985}

Richard Arens, Reducing the order of the Lagrangean for a classical field in curved space-time ................................ 209

Shiferaw Berhanu, W. Wistar (William) Comfort and James Dolan Reid, Counting subgroups and topological group topologies $\ldots \ldots \ldots \ldots \ldots 217$

Michael James Cambern, Isomorphisms of spaces of norm-continuous

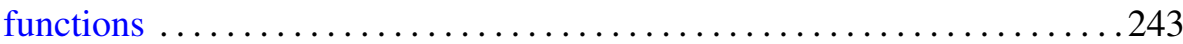

Gerald Arthur Edgar and Jun Feng Zhao, The ordering structure on

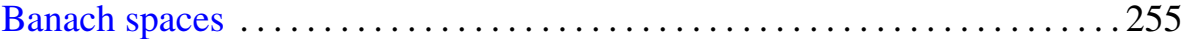

Irving Leonard Glicksberg, An analogue of Liapounoff's convexity theorem for Birnbaum-Orlicz spaces and the extreme points of their unit

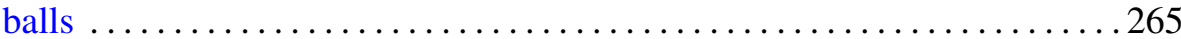

Daniel Heifetz, $p$-adic oscillatory integrals and wave front sets . ........285

José E. Pantoja, Liftings of supercuspidal representations of $\mathrm{Gl}_{2} \ldots \ldots \ldots 307$

Steven C. Pinault, An isoperimetric inequality for surfaces stationary with respect to an elliptic integrand and with at most three boundary

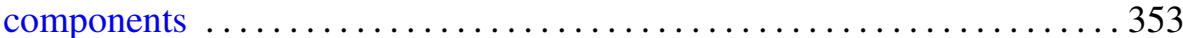

Georges Pinczon, Nonlinear multipliers and applications .............. 359 